\title{
Comment on 'The latency period of mesothelioma among a cohort of British asbestos workers (1978-2005)'
}

\author{
D Mirabelli*,1 and D Zugna ${ }^{1}$ \\ ${ }^{1}$ Cancer Epidemiology Unit, University of Turin, Via Santena 7, 10126 Torino, Italy
}

Sir,

In a paper published on the October 1 issue, Frost (2013) analysed mortality from mesothelioma among members of the Great Britain Asbestos Survey (GBAS), to test whether higher asbestos exposure shortened mesothelioma latency. The author applied accelerated failure time models to estimate time ratios and concluded that there was not sufficient evidence that greater intensity of exposure to asbestos led to shorter latencies. We are concerned that this analysis may have been inappropriate and the conclusions unwarranted.

As from Frost's Table 1, out of 94960 workers entering GBAS between 1978 and 2005-start and, respectively, end of observation-614 died from mesothelioma and 14009 from other causes: the large majority (85\%) were alive at followup and mesothelioma deaths represented $4.4 \%$ of all deaths. In this framework, both (right) censoring and important competitive mortality were present.

As only cohort members dying with mesothelioma-a reasonable proxy for incident cases of mesothelioma-were included, the analysis was limited to the subset of individuals who developed the outcome of interest. Such an approach is at variance with the risk-set sampling designs traditionally used to analyse data from cohort studies (Langholz and Richardson, 2010).

In a competitive risk framework, two key quantities are of interest: the causespecific hazard function $\lambda_{l}(t)$, which can be interpreted approximately as the instantaneous risk per time unit of failure at time $t$ from cause $l$ conditional on survival until just before $t$; and the cumulative incidence function $I_{l}(t)$, which is the probability to fail from cause $l$ before $t$. As $I_{l}(t)$ depends on both the hazard function for cause $l, \lambda_{l}(t)$, and the hazard functions for other competitive causes, there is no one-to-one correspondence between $\lambda_{l}(t)$ and $I_{l}(t)$, that is, the relationships between the explanatory variables and $\lambda_{l}(t)$ may not reflect the relationships between explanatory variables and $I_{l}(t)$ (Andersen et al, 2012).

For simplicity, let $0<t_{1}<t_{2}<\ldots<t_{N}$ be the ordered distinct time points at which failures of any cause occur. In a non-parametric setting, $I_{l}(t)$ can be estimated as follows:

$$
I_{l}(t)=\sum_{j: t_{j} \leqslant t} S\left(t_{j-1}\right) \lambda_{l}\left(t_{j}\right)=\sum_{j: t_{j} \leqslant t}\left[\prod_{j: t_{j} \leqslant t}\left(1-\sum_{l=1}^{k} \frac{d_{l j}}{n_{j}}\right)\right] \frac{d_{l j}}{n_{j}}
$$

where $S\left(t_{j-1}\right)$ is the survival function at time $t_{j-1}, d_{l j}$ is the number of subjects failed from cause $l$ at $t_{j}, n_{j}$ is the number of subjects at risk at $t_{j}$, that is, subjects still in follow-up and not failed from any causes at time $t_{j}$, and $l=1, \ldots, k$ are the competitive causes.

From expression (1), it is clear that restricting the analysis to individuals failing from the cause of interest $l$ will affect both the set risk over time-that is, $n_{j}$ at each time $t_{j}$-and the complement to one of the survival function, $\sum_{l=1}^{k} \frac{d_{l_{j}}}{n_{i}}$, as much as the censoring and the competitive causes are large. Hence estimates of $\lambda_{l}(t)$ and $I_{l}(t)$ will be biased. Again from Frost's Table 1, out of more than 1.6 million person-years of observation in the full cohort, mesothelioma decedents contributed only 9280 person-years.

We want to recall here the scenario depicted by Pike and Doll (1965), who used exposure and mortality data from the British doctors study to argue that practical conditions of human exposure to cigarette smoking, which included consideration of both amount and duration of smoke and competitive mortality, would not lead to significant differences in average age at death from lung cancer between 'heavy' and 'light' smokers. Only much higher exposures, that cannot be encountered in practice, could do the opposite. They concluded that the lack of anticipation in the age of occurrence of lung cancer (as represented by age at death) could not be considered evidence of lack of effect from tobacco smoking: it could at most be interpreted as showing that smoking is not a 'strong' carcinogen-in the meaning used in experimental carcinogenesis. This was their warning against the use of 'life-span', as they called it, or cohort, as we might say, average age at death.

However, their paper included a stronger, introductory remark against the use of another type of average: 'period' average. If the observation period during which cases are enroled in a study is fixed by the observer, when cases of the disease of interest are split into groups according to some 'exposure', their differences in time-dependent variables such as age at death, age at onset and latency since some event (start of exposure, for instance) will not depend on any biological property of the exposure, but solely or predominantly on historical factors: the period when an industry entailing that exposure was developed or phased out is just an example. We think that this second caution applies to GBAS in general, and in particular to Frost's analysis, as it has both left truncation (for cohort members first exposed before 1978) and a closing date at which about $85 \%$ of cohort members were alive. Further, the two main comparison groups, that is, asbestos insulators and asbestos removers, had rather distinct secular trends: basically, when the former occupation started to disappear, the latter started to develop.

We believe that the above remarks are relevant not only for naive statistics, like average time to events such as death or incidence, but also extend properly to the distributions of times to event and therefore to relative times, that can be defined as: 'ratios of times that a given percentage of individuals with different exposures take to develop the event' (Cox et al, 2007). The analysis carried out by Frost did not find evidence of consistent deviations of time ratios from unity because there was no chance for such deviations to occur consistently in the GBAS observational setting - as, indeed, in most if not all other cohort studies.

\section{REFERENCES}

Andersen PK, Geskus RB, de Witte T, Putter H (2012) Competing risks in epidemiology; possibilities and pitfalls. Int J Epidemiol 41: 861-870.

Cox C, Chu H, Schneider MF, Munoz A (2007) Parametric survival analysis and taxonomy of hazard functions for the generalized gamma distribution. Stat Med 26: $4352-4374$

Frost G (2013) The latency period of mesothelioma among a cohort of British asbestos workers (1978-2005). Br J Cancer 109: 1965-1973.

Langholz F, Richardson DB (2010) Fitting general relative risk models for survival time and matched case-control analysis. Am J Epidemiol 171: 377-383.

Pike MC, Doll R (1965) Age at onset of lung cancer: significance in relation to effect of smoking. Lancet 1: 665-668.

*Correspondence: Dr D Mirabelli; E-mail: dario.mirabelli@cpo.it

\section{Neoadjuvant chemotherapy in pancreatic cancer: innovative, but still difficult}

\author{
S Boeck ${ }^{*}, 1$, M Haas ${ }^{1}$, S Ormanns ${ }^{2}$, S Kruger ${ }^{1}$, J T Siveke ${ }^{3}$ and V Heinemann ${ }^{1}$
}

${ }^{1}$ Department of Internal Medicine III and Comprehensive Cancer Center, Klinikum Grosshadern, Ludwig-Maximilians-University of Munich, D-81377 Munich, Germany; ${ }^{2}$ Department of Pathology, Ludwig-Maximilians-University of Munich, D-81377 Munich, Germany and ${ }^{3}$ Department of Internal Medicine II, Klinikum Rechts der Isar, Technical University of Munich, D-81377 Munich, Germany

Sir,

We read with great interest the recent article by Alvarez and co-workers reporting the effects of nab-paclitaxel on tumour stroma in pancreatic cancer (Alvarez et al, 2013). The authors should be congratulated on their interesting findings from a translational study that investigated the biological effects of neoadjuvant gemcitabine and 
nab-paclitaxel in patients with resectable disease. Although the primary study endpoint was to determine the effects of this novel chemotherapy regimen on tumour stroma (including an assessment of collagen content and structure of 'cancer-associated fibroblasts' (CAF)), some important lessons can be learned from this innovative study regarding the approach of neoadjuvant chemotherapy in localised pancreatic cancer (Heinemann et al, 2013).

The investigators enroled 16 patients with histologically or cytologically confirmed resectable or borderline resectable exocrine pancreatic cancer that had received two cycles of gemcitabine/nab-paclitaxel. After chemotherapy, 3 patients had progressive disease and 12 patients were able to proceed to surgical resection. Of note, in the final pathological assessment of the surgical specimen, two study patients in fact had a neuroendorcine cancer and not pancreatic adenocarcinoma. No objective response by RECIST was observed after neoadjuvant chemotherapy; however, half of patients had a CA $19-9$ decrease of $>75 \%$ and a partial metabolic response by FDG-PET imaging. The authors reported a median CA 19-9 pre-treatment value of $2.588 \mathrm{U} / \mathrm{dl}$ in their patient population, ranging from 1 to $36.376 \mathrm{U} / \mathrm{dl}$; this would correspond to a median CA 19 9 level of $26 \mathrm{U} \mathrm{ml}^{-1}$ at baseline with a range from $0.01 \mathrm{U} \mathrm{ml}^{-1}$ to 364 $\mathrm{U} \mathrm{ml}^{-1}$, respectively (please note: $(\mathrm{U} / \mathrm{dl})$ is a very uncommon unit for reporting CA 19-9; the most commonly used unit in the literature is $\left(\mathrm{U} \mathrm{ml}^{-1}\right)$ ) (Boeck et al, 2006). On the basis of the evidence from previous CA 19-9 biomarker studies, significantly elevated CA 19-9 levels before surgical resection may indicate a potential subradiographic systemic disease rather than a localised disease. In a single-centre trial from the USA, 51 out of 262 patients who underwent staging laparoscopy for radiographically resectable pancreatic adenocarcinoma in fact had unresectable disease. In this study, the median pre-operative CA 19-9 value for patients who underwent resection was $131 \mathrm{Uml}^{-1}$ vs $379 \mathrm{U} \mathrm{ml}^{-1}$ for those patients with unresectable disease $(P=0.003)$. With the use of a receiver operating characteristics curve for pre-operative CA 19-9 value and tumour resectability, the statistically optimal cut-off value was determined to be $130 \mathrm{U} \mathrm{ml}^{-1}$ (Maithel et al, 2008). In concordance, Katz et al (2010) suggested a pre-treatment CA 19-9 cut-off of 149 $\mathrm{U} \mathrm{ml}^{-1}$ as a predictor of completing chemoradiation and subsequent pancreaticoduodenectomy in their neoadjuvant study (Ketz et al, 2010). In the light of the fact that '.. the content of stroma and CAF may change with different cancer stages' (as the authors themselves stated correctly in the Discussion section), one should keep in mind that an appropriate selection of patients with a unique stage of disease thus should be an important issue in novel translational studies.

Nevertheless, the data presented are important and highlight an innovative approach to how future translational research should be conducted in pancreatic cancer. However, from a clinical point of view, this small study clearly unbosoms potential pitfalls of neoadjuvant treatment approaches in resectable pancreatic cancer: (1) the difficulty of adequately select patients with a real localised disease, (2) the limitations of obtaining an adequate pre-treatment histological diagnosis and (3) the often missing objective response by RECIST to neoadjuvant chemotherapy. The latter one was already described previously in borderline resectable patients and in fact it may be more appropriate to investigate different clinical methods than conventional CT scan for assessing treatment response to a neoadjuvant treatment regimen (e.g. metabolic response by PET or biochemical response by CA 19-9) (Takahashi et al, 2010; Katz et al, 2012).

We thus would recommend including a rigorous histological (no cytological) confirmation of disease before study entry, an assessment of response by different methods other than CT scan and also a CA 19-9 cut-off for patients entering neoadjuvant trials. Such an approach has recently been included in several large phase III adjuvant trials (e.g. RTOG 0848, Prodige 24/Accord 24), mainly by using a post-resection CA 19-9 level of $<180 \mathrm{U} \mathrm{ml}^{-1}$ as an inclusion criterion.

\section{REFERENCES}

Alvarez R, Musteanu M, Garcia-Garcia E, Lopez-Casas PP, Megias D, Guerra C, Muñoz M, Quijano Y, Cubillo A, Rodriguez-Pascual J, Plaza C, de Vicente E, Prados S, Tabernero S, Barbacid M, Lopez-Rios F, Hidalgo M (2013) Stromal disrupting effects of nab-paclitaxel in pancreatic cancer. Br J Cancer 109: 926-933.

Boeck S, Stieber P, Holdenrieder S, Wilkowski R, Heinemann V (2006) Prognostic and therapeutic significance of carbohydrate antigen 19-9 as tumor marker in patients with pancreatic cancer. Oncology 70: 255-264.

Heinemann V, Haas M, Boeck S (2013) Neoadjuvant treatment of borderline resectable and non-resectable pancreatic cancer. Ann Oncol 24: 2484-2492.

Katz MH, Fleming JB, Bhosale P, Varadhachary G, Lee JE, Wolff R, Wang H, Abbruzzese J, Pisters PW, Vauthey JN, Charnsangavej C, Tamm E,

Crane CH, Balachandran A (2012) Response of borderline resectable pancreatic cancer to neoadjuvant therapy is not reflected by radiographic indicators. Cancer 118: 5749-5756.

Katz MH, Varadhachary GR, Fleming JB, Wolff RA, Lee JE, Pisters PW, Vauthey JN, Abdalla EK, Sun CC, Wang H, Crane CH, Lee JH, Tamm EP, Abbruzzese JL, Evans DB (2010) Serum CA 19-9 as a marker of resectability and survival in patients with potentially resectable pancreatic cancer treated with neoadjuvant chemoradiation. Ann Surg Oncol 17: 1794-1801.

Maithel SK, Maloney S, Winston C, Gönen M, D’Angelica MI, Dematteo RP, Jarnagin WR, Brennan MF, Allen PJ (2008) Preoperative CA 19-9 and the yield of staging laparoscopy in patients with radiographically resectable pancreatic adenocarcinoma. Ann Surg Oncol 15: 3512-3520.

Takahashi H, Ohigashi H, Ishikawa O, Eguchi H, Gotoh K, Yamada T, Nakaizumi A, Uehara H, Tomita Y, Nishiyama K, Yano M (2010) Serum CA19-9 alterations during preoperative gemcitabine-based chemoradiation therapy for resectable invasive ductal carcinoma of the pancreas as an indicator for therapeutic selection and survival. Ann Surg 251: 461-469.

\title{
Stellate cells, a point of light in the dark night of pancreatic cancer
}

\section{N Ramírez ${ }^{*}, 1$, A Viúdez ${ }^{2}$, I Hernández-García ${ }^{*}, 2$, D Guerrero ${ }^{3}$, M Gómez-Dorronsoro ${ }^{4}$, F J Herrera ${ }^{5}$, J Vila ${ }^{6}$, L Beloki ${ }^{1}$, M Ciaúrriz $^{1}$, C Mansilla ${ }^{1}$ and R Vera ${ }^{2}$}

\begin{abstract}
${ }^{1}$ Oncohematology Research Group, Navarrabiomed-Miguel Servet Foundation, Pamplona, Spain; ${ }^{2}$ Department of Oncology, Complejo Hospitalario de Navarra, 31008 Pamplona, Spain; ${ }^{3}$ Cancer Epigenetics Group, Navarrabiomed-Miguel Servet Foundation, Pamplona, Spain; ${ }^{4}$ Department of Pathology, Complejo Hospitalario de Navarra, Pamplona, Spain; ${ }^{5}$ Department of Surgery, Complejo Hospitalario de Navarra, Pamplona, Spain and ${ }^{6}$ Endoscopy Unit, Gastroenterology Department, Complejo Hospitalario de Navarra, Pamplona, Spain
\end{abstract}

Sir,

We have read with particular attention the recent article published in the BJC by Alvarez et al (2013).

They, through endoscopic ultrasound elastography system and conventional immuno-histochemical assays, perform an intrinsic characterisation of tumour stroma's stiffness, fibroblast density and architecture focusing in type I collagen fibre arrangement, cancer-associated fibroblasts (CAFs) and activated CAF quantification. The analysis of post-treatment tissues from patients treated with nab-paclitaxel and gemcitabine combination showed a decrease in CAF number and changes in collagen architecture.
These results reaffirm the potential ability of nab-paclitaxel to target the stroma and to change its phenotype, altering the 'hard of the rock', as described by Garber (2010), intensifying its permeability to deliver cytotoxic agents such as gemcitabine by increasing tumour vascularisation or enzymatic inhibition (Frese et al, 2012) that would suppose a better pharmacokinetic profile (Von Hoff et al, 2011). Even though it is known the clinical benefit of nab-paclitaxel plus gemcitabine in metastatic pancreatic cancer, the cellular or molecular mechanisms that are expressly addressing this stromal involution have not been appointed yet (Alvarez et al, 2013). In this sense, authors analysed the role of secreted protein, acidic and rich in 